Herizo Rakotovololonalimanana ${ }^{1,5,6}$ HerY RAKOTONDRAOELINA 3,7

Gilles ChaIX ${ }^{2,6}$

Lolona RAMAMONJISOA 4

Marie-France THEVENON ${ }^{5}$

Honoré RANDRIANJAFY 3,7

Tahiana RAMANANANTOANDRO ${ }^{1}$

1 Université d'Antananarivo

École supérieure des sciences

agronomiques

Départements des eaux et forêts

BP 175, Antananarivo 101

Madagascar

2 Universidade de São Paulo

Departamento de Ciências Florestais

da ESALQ

Avenida Pádua Dias 11

Caixa Postal 9

CEP 13418-900

Piracicaba-SP

Brésil

${ }^{3}$ Drfp-Fofifa

BP 904

Antananarivo 101

Madagascar

${ }^{4}$ Silo national des graines forestières

BP 5091

101 Antananarivo

Madagascar

${ }^{5}$ Cirad

UR BioWooEB

TA B 114/16

34398 Montpellier Cedex 5

France

${ }^{6}$ Cirad

Umr Agap

Avenue Agropolis

34398 Montpellier Cedex 5

France

7 Cirad

DP Forêts et Biodiversité BP 853, Antananarivo

Madagascar

\section{Adaptation et variabilité génétique de la croissance de Liquidambar styraciflua $L$. de l'essai de provenances à Mandraka, Madagascar}

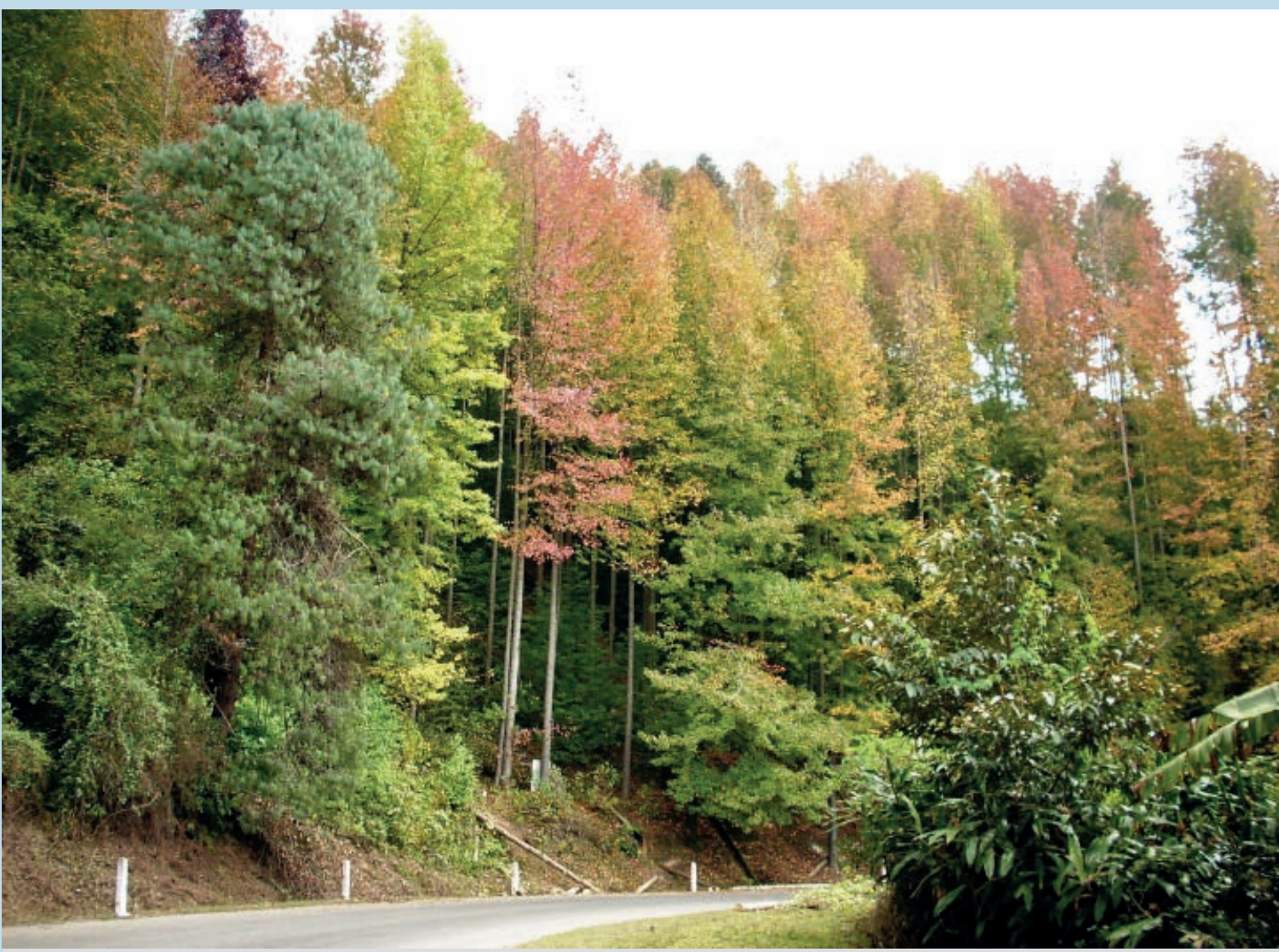

Photo 1.

Liquidambar styraciflua âgé de 22 ans de l'essai de provenances à Mandraka. 


\section{RÉSUMÉ}

\section{ADAPTATION ET VARIABILITÉ GÉNÉTIQUE DE LA CROISSANCE DE LIQUIDAMBAR STYRACIFLUA L. DE L'ESSAI DE PROVENANCES À MANDRAKA, MADAGASCAR}

Liquidambar styraciflua ou copalme d'Amérique est une espèce feuillue originaire $d u$ Sud-Est des États Unis d'Amérique et de l'Amérique Centrale. L'espèce possède un comportement remarquable en plantation et les propriétés de son bois en font un matériau à usage multiples. En 1986, dans le cadre d'un essai international de provenances de Liquidambar styraciflua, le Commonwealth Forestry Institute (CFI) d'Oxford a diffusé dans plusieurs pays (Brésil, Mexique, Madagascar) des graines de treize provenances récoltées essentiellement dans l'aire de distribution naturelle de l'espèce dans l'Est des États-Unis d'Amérique, du Mexique et de l'Amérique centrale. À Madagascar, le Département des Recherches Forestières et Piscicoles (DRFP-FOFIFA) les a plantées à Mandraka (Madagascar). Des données annuelles de la croissance ont été collectées durant 25 ans ; cependant les résultats n'ont pas encore été publiés contrairement à ceux du Brésil et du Mexique. Il s'agit ici d'évaluer l'adaptation de l'essence à Mandraka et d'étudier la variabilité intraspécifique de sa croissance. Jusqu'à 25 ans, la croissance de l'espèce est régulière pour atteindre en moyenne $26 \mathrm{~m}$ en hauteur, $38 \mathrm{~cm}$ en diamètre et $0,12 \mathrm{~m}^{2} /$ arbre en surface terrière. Les valeurs moyennes des provenances pour ces mêmes caractéristiques et la surface terrière sont significativement différentes. L'héritabilité au sens large à 25 ans est de 0,76 , 0,60 et 0,63 respectivement pour la hauteur, le diamètre et la surface terrière, montrant un contrôle génétique de la croissance. Dans des conditions écologiques similaires et à âges équivalents, la croissance de $L$. styraciflua est équivalente à celle des espèces du genre Eucalyptus et supérieure à celle des Pinus. Par rapport aux essais internationaux du CFI, l'essai de provenances de L. styraciflua dans le site de la Mandraka montre une meilleure adaptation et une bonne croissance de l'espèce.

Mots-clés : Liquidambar styraciflua, caractères de croissance, adaptation, essai de provenances, Madagascar.

\section{ABSTRACT}

\section{ADAPTATION AND GENETIC VARIABILITY IN THE GROWTH OF LIQUIDAMBAR STYRACIFLUA L. FROM THE PROVENANCE TRIAL AT MANDRAKA, MADAGASCAR}

Liquidambar styraciflua, or the American sweet-gum tree, is a deciduous species originating in the south-eastern United States and Central America. Its behaviour in plantations is outstanding and its timber is used for multiple purposes. In 1986, under an international provenance trial, the Commonwealth Forestry Institute (CFI) in Oxford distributed Liquidambar styraciflua seeds of thirteen different provenances, mainly harvested in the natural range of distribution of the species extending from the eastern United States to Mexico and Central America. In Madagascar, they were planted by the forestry and fisheries research department (DRFP-FOFIFA) at Mandraka. Data on annual growth were collected for 25 years, but the results, unlike those for Brazil and Mexico, have not yet been published. The aim of our study was to assess adaptation of the species in Mandraka and to investigate intra-species growth variability. Up to 25 years of age, the trees grew steadily to reach, on average, a height of $26 \mathrm{~m}$, a diameter of $38 \mathrm{~cm}$ and a basal area of $0.12 \mathrm{~m}^{2}$ per tree. These average values differ significantly according to provenance. At 25 years of age, heritability in the broad sense is $0.76,0.60$ and 0.63 for height, diameter and basal area respectively, indicating genetic control over growth. Under similar ecological conditions and at equivalent age, the growth of L. styraciflua is equivalent to that of species of the genus Eucalyptus and better than in Pinus. In comparison with the international CFI trials, the provenance trial for L. styraciflua at the Mandraka site shows better adaptation and a high growth rate for the species.

Keywords: Liquidambar styraciflua, growth traits, adaptation, provenance trial, Madagascar.

\section{RESUMEN}

\section{ADAPTACIÓN Y VARIABILIDAD GENÉTICA DEL CRECIMIENTO DE LIQUIDAMBAR STYRACIFLUA L. EN EL ENSAYO DE PROCEDENCIAS DE MANDRAKA, MADAGASCAR}

Liquidambar styraciflua, o árbol del ámbar, es una frondosa originaria del sudeste de Estados Unidos y América Central. Esta especie presenta un excelente comportamiento en siembra y su madera tiene múltiples usos. En 1986, en el marco de un ensayo internacional de procedencias de Liquidambar styraciflua, el Commonwealth Forestry Institute (CFI) de Oxford difundió en varios países (Brasil, México y Madagascar) semillas de trece procedencias cosechadas fundamentalmente en el área de distribución natural de esta especie: el este de Estados Unidos, México y América Central. En Madagascar, el Département des Recherches Forestières et Piscicoles (DRFP-FOFIFA) las plantó en Mandraka (Madagascar). Durante 25 años se recopilaron los resultados anuales de crecimiento; sin embargo, y al contrario de los de Brasil y México, los resultados aún no han sido publicados. El objetivo aquí es evaluar la adaptación de la especie en Mandraka y estudiar la variabilidad interespecífica de su crecimiento. Hasta los 25 años el crecimiento de la especie es regular, alcanzando $26 \mathrm{~m}$ de altura promedio, $38 \mathrm{~cm}$ de diámetro y $0.12 \mathrm{~m}^{2} /$ árbol de área basal. Los valores promedio de las procedencias para estas mismas características, así como su área basal, presentan diferencias significativas. La heredabilidad en sentido amplio a los 25 años es de $0.76,0.60$ y 0.63 para la altura, el diámetro y el área basal, respectivamente; lo que muestra un control genético del crecimiento. En condiciones ecológicas análogas y con edades equivalentes, el crecimiento de $L$. styraciflua es similar al de las especies del género Eucalyptus y superior al de Pinus. Con respecto a los ensayos internacionales del $\mathrm{CFI}$, el ensayo de procedencias de L. styraciflua en la estación de la Mandraka muestra una mejor adaptación y un buen crecimiento de la especie.

Palabras clave: Liquidambar styraciflua, caracteres de crecimiento, adaptación, ensayo de procedencias, Madagascar. 


\section{Introduction}

Liquidambar styraciflua L. ou copalme d'Amérique fait partie des espèces feuillues les plus importantes des forêts du Sud-Est des États-Unis d'Amérique, avec un stock total de bois de liquidambar estimé à plus de 13 millions de $\mathrm{m}^{3}$ (FAO, 2007 ; WRIGHT, CUNNINGHAM, 2008). Cette espèce possède une très grande tolérance pour les différents types de sols, avec une prédilection pour les sols profonds, acides et bien arrosés (WRIGHT, CUNNINGHAM, 2008). L. styraciflua est depuis longtemps utilisé comme essence de reboisement, notamment en Amérique latine (SABISTINA, 2010). Son bois est largement valorisé, tant en pâte à papier, palettes ou bois de charpente dans le Sud des ÉtatsUnis (WRIGHT, CUNNINGHAM, 2008) qu'en contreplaqués, emballages légers, caisses renforcées en bois déroulé, menuiserie, agencement intérieur décoratif, tournerie, ameublement sous forme de bois massif ou placage sur tout le territoire américain (ALDEN, 1995 ; SABISTINA, 2010). RAKOTONDRAOELINA et RAKOTOVAO (2005) ont par ailleurs rapporté que le liquidambar a été régulièrement importé des États-Unis vers l'Europe comme substitut du noyer, après coloration, pour les meubles de série en bois massif. Ainsi, cette essence montre des potentialités d'introduction et de valorisations multiples.

L. styraciflua a été introduit à Madagascar dans les années 1950 (FOFIFA, 1990) dans différents arboretums et stations forestières (figure 1). En 1986, un essai international de provenances de L. styraciflua a été initié par le Commonwealth Forestry Institute (Cfi) d'Oxford. À Madagascar, le site de Mandraka a été retenu, ainsi que cinq autres localités du continent sud-américain : Xalapa (Mexique), Agudos, Iguaçu, Paraguaçu, Colombo (Brésil). Des données annuelles de la croissance ont été collectées sur le site de Mandraka ; cependant, aucun article n'a été publié pour les essais malgaches, exceptés les rapports annuels du Département de recherches forestières et piscicoles (Drfp) du Fofifa (Centre national de la recherche appliquée au développement rural), contrairement aux sites mexicains et brésiliens pour lesquels les auteurs cités ci-après ont publié de nombreux résultats (SHIMIZU, SPIR, 1999 ; MATTOS et al., 2001 ; SHIMIZU, SPIR, 2002 ; SEBBENN et al., 2007 ; MENDIZABAL-HERNANDEZ et al., 2009). Aussi ce travail se propose-t-il d'évaluer l'adaptation de cette espèce à Mandraka en étudiant sa croissance après 25 ans de suivi et la variabilité intraspécifique par rapport aux autres espèces introduites, largement répandues et utilisées à Madagascar (Pinus et Eucalyptus), et aux résultats publiés des autres essais internationaux.

\section{Matériels et méthodes}

\section{Matériel végétal}

Le matériel d'étude est constitué par treize provenances de Liquidambar styraciflua issues de cinq pays de l'aire naturelle (figure 2) et introduites à Mandraka en 1986 (tableau I, photo 1). Le site d'essai à Mandraka (figure 1) se trouve à $67 \mathrm{~km}$ à l'Est de la capitale, Antananarivo (longitude : 4755'E, latitude : $18^{\circ} 54^{\prime}$ 'S, altitude : $1200 \mathrm{~m}$, température moyenne : $17,5^{\circ} \mathrm{C}$, pluviosité annuelle moyenne : $2300 \mathrm{~mm}$ ). L'expérimentation a été mise en place sur un terrain vallonné caractéristique de la région, dont la pente maximale peut atteindre $80 \%$ à $90 \%$. Avant la plantation, le terrain était recouvert d'une maigre forêt secondaire dominée principalement par les espèces telles que harongana,

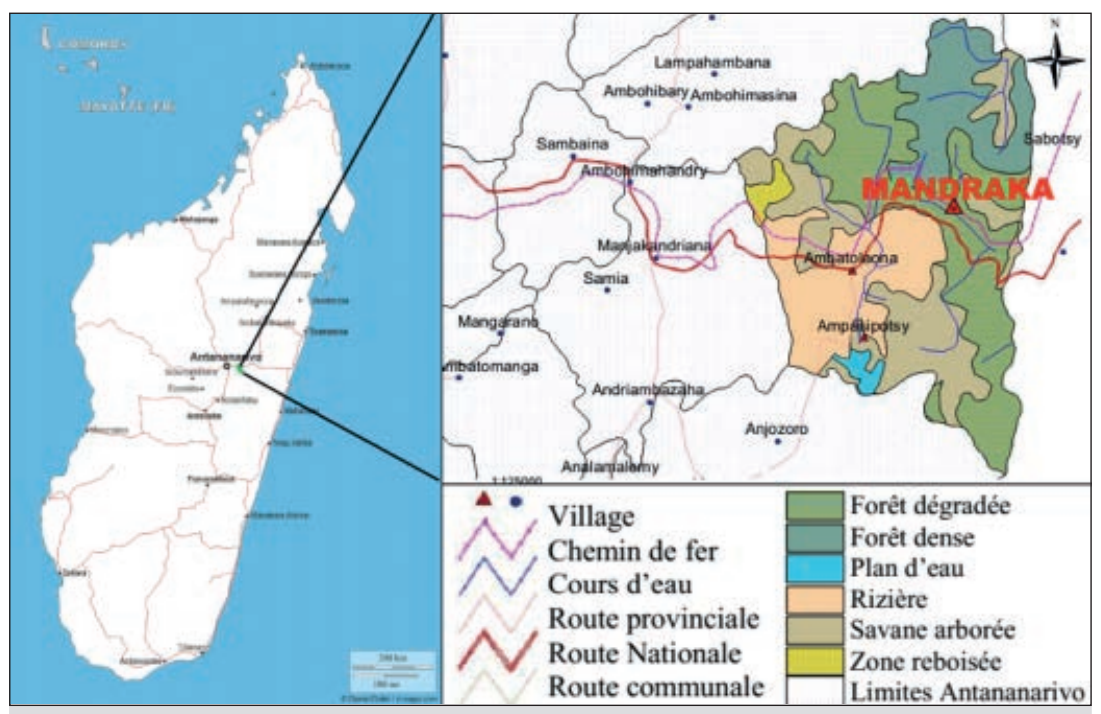

Figure 1.

Localisation de l'essai de provenances de Liquidambar styraciflua à Madagascar.

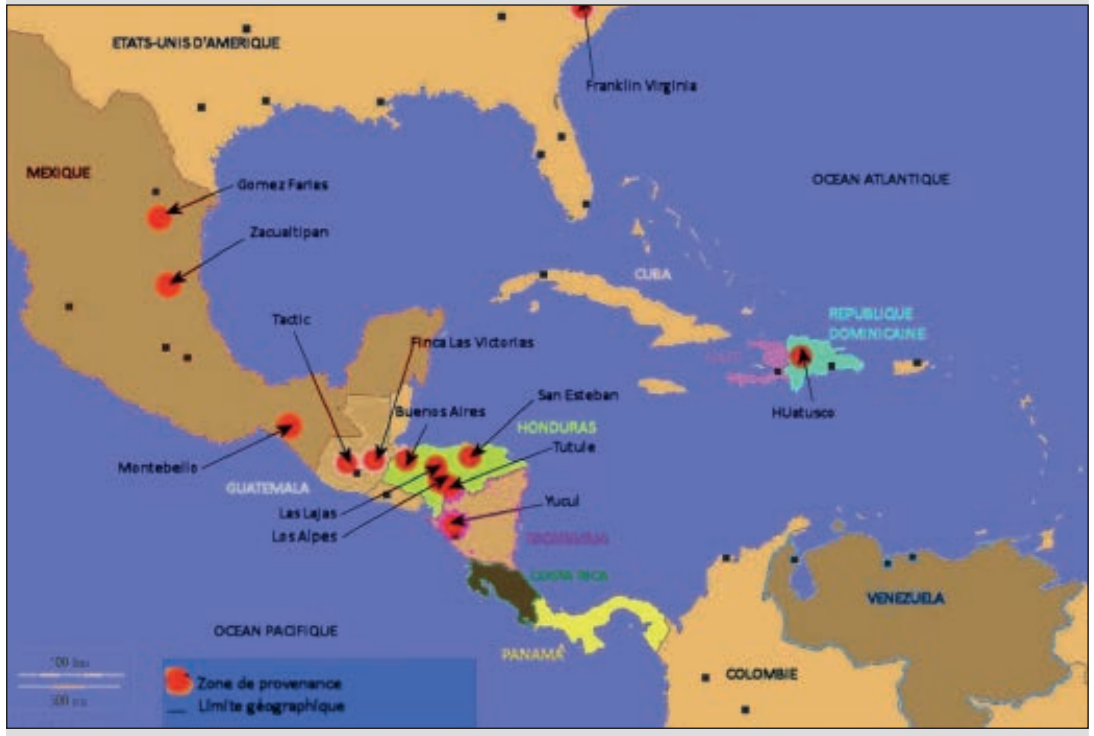

Figure 2.

Localisation dans l'aire naturelle des provenances de

Liquidambar styraciflua testées dans l'essai de Mandraka. 
Harungana madagascariensis, lalona, Weinmannia spp., et hafotra, Dombeya sp. Après défrichement, une trouaison a été effectuée $(80$ x $80 \mathrm{~cm})$ sur les banquettes situées en courbes de niveau. Le dispositif expérimental est constitué par quatre blocs complets randomisés constitués de 13 parcelles unitaires ( 1 par provenance) de 36 plants $(3 \times 3)$ réparties aléatoirement dans chacun des blocs. L'écartement initial de plantation était de $2,5 \times 2,5 \mathrm{~m}$, soit une densité de plantation de 1600 tiges/ha. La plantation a fait l'objet d'une éclaircie sanitaire avec un taux de prélèvement de $30 \%$ à 10 ans et une éclaircie sélective de $42 \%$ à 17 ans. Suite à ces deux éclaircies, l'essai de provenances ne comptait plus que 465 pieds (ce qui équivaut à environ 11 pieds par provenance sur les 36 intialement), soit une densité de 310 tiges/ha en moyenne. Une éclaircie sélective partielle supplémentaire de $30 \%$ a été réalisée sur les arbres du bloc I à 23 ans (2009), et sur ceux du bloc III à 24 ans (2010). Par conséquent, le site ne contenait plus que 411 pieds (soit 274 tiges/ha en moyenne) à partir de 24 ans.

\section{Paramètres considérés}

L'évaluation de la croissance de l'espèce repose sur les inventaires annuels des douze provenances réalisés par le Drfp-Fofifa de la hauteur totale (années 1992 à 1999) et du diamètre à 1,30 m (années 1992 à 2008). La surface terrière individuelle de chaque pied a également été considérée. La fermeture de la canopée rendait difficile le relevé de la hauteur après 1999, mais, à la suite des éclaircies réalisées, ce paramètre a pu être mesuré sur neuf des douze provenances en 2011, c'est-à-dire 25 ans après la plantation. Les neuf provenances ont été choisies sur la base des valeurs moyennes des hauteurs et des diamètres les plus élevées L'effet de l'éclaircie a été évalué trois ans après chaque éclaircie, c'est-à-dire à 13 ans (1999), à 20 ans (2006) et à 25 ans (2011) après plantation. Pour la comparaison avec les autres essais internationaux du Cfi et les résultats publiés, il a été considéré les données disponibles à $9,11,16$ et 19 ans et les plus proches de notre étude.

\section{Méthode d'analyse}

Afin d'utiliser les analyses statistiques appropriées, la normalité de la distribution de la hauteur, du diamètre et de la surface terrière a été étudiée avec le test de Shapiro-Wilk et l'homogénéité des variances de ces paramètres avec le test de Bartlett (MOUCHIROUD, 2003 ; SAPORTA, 2006). Ces vérifications réalisées et concluantes, une analyse de la variance a été effectuée pour comparer les moyennes par provenances. Dans le cas contraire, le test de KruskalWallis, adapté à ce contexte, a été effectué.

Pour l'analyse de la variance, le modèle statistique est le suivant (formule 1) :

$Y_{i j}=\mu+B_{i}+P_{j}+(B P)_{i j}+\varepsilon_{i j}$

avec :

$i=1, \ldots . . .$. I I avec / le nombre de blocs ;

$j=1, \ldots . . . .$. J J avec $/$ le nombre de provenances ;

$Y_{i j}$ : valeur phénotypique mesurée sur l'individu de provenance $j$ sur le bloc $i$;

$\mu$ : moyenne générale ;

$B_{i}$ : effet du bloc de répétition avec comme variance $\sigma_{\mathrm{B}}^{2}$;

$P_{j}$ : effet de la provenance avec comme variance $\sigma^{2}{ }_{\mathrm{P}}$;

$B P_{i j}$ : effet de l'interaction bloc x provenance avec comme variance $\sigma^{2}$ BP ;

$\varepsilon_{\mathrm{ij}}$ : résidus à effet aléatoire de variance $\sigma^{2} \varepsilon$.

Tableau I.

Caractéristiques des provenances de Liquidambar styraciflua introduites à Mandraka.

\begin{tabular}{|c|c|c|c|c|c|c|c|}
\hline $\mathbf{N}^{\circ}$ & Provenance & Origine & Longitude & Latitude & $\begin{array}{l}\text { Altitude } \\
\text { (m) }\end{array}$ & $\begin{array}{c}\mathrm{T}^{\circ} \text { moy } \\
\left({ }^{\circ} \mathrm{C}\right)\end{array}$ & $\begin{array}{l}\text { Pluviométrie } \\
(\mathrm{mm})\end{array}$ \\
\hline 1 & Franklin, Virginia & USA & $76^{\circ} 55^{\prime} \mathrm{W}$ & $36^{\circ} 40^{\prime} \mathrm{N}$ & 548 & 13 & 940 \\
\hline 2 & Gomez Farias & Mexico & $99^{\circ} 10^{\prime} \mathrm{W}$ & $23^{\circ} 06^{\prime} \mathrm{N}$ & $950-1450$ & 17,0 & $1800-2500$ \\
\hline 3 & Zacualtipan & Mexico & 98³7’W & $20^{\circ} 42^{\prime} \mathrm{N}$ & $1800-1870$ & 13,6 & 2047 \\
\hline 4 & Huatusco & Mexico & $69^{\circ} 58^{\prime} W$ & $19^{\circ} 05^{\prime} \mathrm{N}$ & $1350-1550$ & 18,8 & 1746 \\
\hline 5 & Buenos Aires & Honduras & $88^{\circ} 11^{\prime} W$ & $15^{\circ} 30^{\prime} \mathrm{N}$ & $900-1080$ & 25,0 & 2000 \\
\hline 6 & Montebello & Mexico & $91^{\circ} 44^{\prime} W$ & $16^{\circ} 04^{\prime} \mathrm{N}$ & 1500 & 18,0 & 1836 \\
\hline 7 & Tactic & Guatemala & $90^{\circ} 21^{\prime} \mathrm{W}$ & $15^{\circ} 19^{\prime} \mathrm{N}$ & $1380-1420$ & 17,3 & 2079,5 \\
\hline 8 & Finca las Victorias & Guatemala & $89^{\circ} 22^{\prime} \mathrm{W}$ & $15^{\circ} 12^{\prime} \mathrm{N}$ & $800-1000$ & 22,4 & 2000 \\
\hline 9 & Las Lajas & Honduras & $87^{\circ} 34^{\prime} W$ & $14^{\circ} 48^{\prime} \mathrm{N}$ & $1100-1200$ & 21,4 & 1700 \\
\hline 10 & San Esteban & Honduras & $85^{\circ} 35^{\prime} W$ & $15^{\circ} 22^{\prime} \mathrm{N}$ & $640-800$ & 21,0 & $1300-1500$ \\
\hline 11 & Tutule & Honduras & $87^{\circ} 50^{\prime} W$ & $14^{\circ} 12^{\prime} \mathrm{N}$ & $1400-1700$ & 17,0 & 1900 \\
\hline 12 & Los Alpes & Honduras & $87^{\circ} 58^{\prime} w$ & $14^{\circ} 33^{\prime} \mathrm{N}$ & $1250-1350$ & 18,0 & $1400-1600$ \\
\hline 13 & Yucul & Nicaragua & $85^{\circ} 48^{\prime} \mathrm{W}$ & $12^{\circ} 55^{\prime} \mathrm{N}$ & $850-1100$ & 22,4 & 1394 \\
\hline
\end{tabular}


Tableau II.

Tableau d'analyse de la variance.

Sources

Degré de liberté (ddl)

Carrés moyens (CM)

$\mathrm{i}-1$

Provenances

Blocs

$\mathrm{j}-1$

Blocs x provenance

Résidus

$(i-1)(j-1)$

$\mathrm{ij}(\mathrm{N}-1)$
F calculée

A $\quad A / D$

C

D

$B$

$\begin{array}{ll}B / D & \sigma^{2}{ }_{B} \\ C / D & \sigma^{2}{ }_{B P} \\ & \sigma^{2}{ }_{e}\end{array}$

Estimations des CM

$\mathrm{F}$ : valeur de la variable de Fischer-Snedecor de l'analyse de la variance ; i : nombre de blocs ; $j$ : nombre de provenances ; $\mathrm{N}$ : nombre d'arbres; $\sigma_{\mathrm{B}}^{2}$ : variance de l'effet du bloc de répétition; $\sigma^{2}{ }_{\mathrm{P}}$ : variance de l'effet de la provenance ;

$\sigma_{\mathrm{BP}}^{2}$ : variance de l'effet d'interaction bloc x provenance ; $\sigma_{\mathrm{e}}^{2}$ : variance des résidus à effet aléatoire.

La méthode de Holm a été ensuite appliquée pour chaque variable afin de détecter les différences significatives entre provenances deux à deux (SAPORTA, 2006 ; AMBROISE, 2007).

L’héritabilité au sens large ou héritabilité génotypique $\left(h_{G}^{2}\right)$ a été estimée à partir de l'analyse de la variance (tableau II) et selon la formule de NANSON (1970) (formule 2) :

$h^{2}{ }_{G}=\frac{\mathrm{A}-\mathrm{D}}{\mathrm{A}}=1-\frac{1}{\mathrm{~F}}$

Ce dispositif n'est pas le plus adéquat pour l'estimation de l'héritabilité des caractères (FALCONER et MACKAY, 1996), mais la formule retenue est la plus adaptée (NANSON, 1970). L'héritabilité renseigne sur la part de variance transmise par les parents à leurs descendants pour le caractère considéré par multiplication générative. Elle permet alors d'indiquer le niveau du contrôle génétique du caractère étudié.

Toutes les analyses ont été exécutées avec le logiciel $\mathrm{R}$, version 2.15.3 (R DEVELOPMENT CORE TEAM, 2012).

\section{Résultats}

\section{Croissance de Liquidambar styraciflua de l'essai de provenances de Mandraka}

À 13 ans, la hauteur moyenne de L. styraciflua à Mandraka est de 19,5 m avec un accroissement moyen annuel de $1,5 \mathrm{~m}$. À 25 ans, la hauteur moyenne est de 26,1 m avec un accroissement moyen annuel de l'ordre de un mètre. Le diamètre moyen de l'essai est de $21,8 \mathrm{~cm}, 31,9 \mathrm{~cm}$ et $38,4 \mathrm{~cm}$ respectivement à 13,20 et 25 ans, soit des accroissements moyens annuels de 1,7, 1,6 et $1,5 \mathrm{~cm}$ (tableau III). Ainsi, l'accroissement en diamètre reste assez stable entre 13 et 25 ans alors que celui en hauteur diminue plus fortement. Le seuil d'exploitabilité des bois d'œuvre de plantation régi par la législation forestière malgache (diamètre supérieur à $30 \mathrm{~cm}$, décret n 98-782 du 16 septembre 1998) (MINISTÈRE DE L'ENVIRONNEMENT, DES EAUX ET FORÊTS, 1998) est atteint à partir de 20 ans. Cela signifie que, dans un même contexte environnemental et sylvicole, il est possible d'établir un plan d'exploitation avec une révolution de 20 ans pour le cas de $L$. styraciflua.

En 2011, lors de l'inventaire et de l'éclaircie du bloc III, des rejets de souches (photo 2) et la présence de régénérations naturelles ont été observés. L'aptitude à rejeter permet d'envisager la gestion de Liquidambar en taillis ou en taillis sous futaie.

Après l'éclaircie de 1999 (10 ans), la surface terrière moyenne était de $22,6 \mathrm{~m}^{2} / \mathrm{ha}$ avec une densité de 570 tiges/ha. Ensuite, après la deuxième éclaircie de 2003 (17 ans), la surface terrière moyenne à 20 ans était de $25,8 \mathrm{~m}^{2} /$ ha avec une densité de peuplement de 310 tiges/ha. Enfin, en 2011 (25 ans), et après la troisième éclaircie des blocs I et III, la surface terrière moyenne du $L$. styraciflua de l'essai de provenances était de $33 \mathrm{~m}^{2} / \mathrm{ha}$ avec une densité de 274 tiges/ha (tableau III). Ces valeurs indiquent une bonne adaptation de l'espèce du point de vue de sa croissance et de la production de bois en volume. L'évolution des valeurs de la surface terrière après les éclaircies montre que le liquidambar réagit bien aux éclaircies (tableau III). 
Tableau III.

Caractéristiques dendrométriques générales et par provenance du Liquidambar styraciflua de l'essai de Mandraka.

\begin{tabular}{|c|c|c|c|c|c|c|c|c|c|c|c|c|c|c|}
\hline \multirow[t]{6}{*}{$\mathbf{N}^{\circ}$} & Paramètre & H & Amah & D & Amad & G & D & Amad & G & H & Amah & D & Amad & G \\
\hline & Année & 1999 & 1999 & 1999 & 1999 & 1999 & 2006 & 2006 & 2006 & 2011 & 2011 & 2011 & 2011 & 2011 \\
\hline & Âge (an) & 13 & 13 & 13 & 13 & 13 & 20 & 20 & 20 & 25 & 25 & 25 & 25 & 25 \\
\hline & Unité & $\mathrm{m}$ & m/an & $\mathrm{cm}$ & $\mathrm{cm} / \mathrm{an}$ & $\mathrm{cm}^{2} /$ arbre & $\mathrm{cm}$ & $\mathrm{cm} / \mathrm{an}$ & $\mathrm{cm}^{2} /$ arbre & $\mathrm{m}$ & $\mathrm{m} / \mathrm{an}$ & $\mathrm{cm}$ & $\mathrm{cm} / \mathrm{an}$ & $\mathrm{cm}^{2} /$ arbre \\
\hline & Nombre d'arbres & 854 & 854 & 854 & 854 & 854 & 465 & 465 & 465 & 305 & 305 & 305 & 305 & 305 \\
\hline & $\begin{array}{l}\text { Densité } \\
\text { (tiges/ha) }\end{array}$ & 577 & 577 & 577 & 577 & 577 & 310 & 310 & 310 & 274 & 274 & 274 & 274 & 274 \\
\hline 2 & Gomez Farias & $18,5^{b c}$ & $1,43^{\mathrm{ab}}$ & $20,6^{b}$ & $1,59^{b}$ & $360^{b c}$ & $31,5^{\mathrm{abcd}}$ & $1,58^{a b c}$ & $896^{a b c}$ & - & - & - & - & - \\
\hline 3 & Zacualtipan & $13,3^{d}$ & $1,02^{c}$ & $16,5^{c}$ & $1,27^{c}$ & $243^{c}$ & $26,5^{d}$ & $1,32^{d}$ & $569^{d}$ & - & - & - & - & - \\
\hline 4 & Huatusco & $18^{c}$ & $1,31^{b}$ & $20^{b}$ & $1,54^{b}$ & $338^{b c}$ & $30,3^{b c d}$ & $1,52^{a b c}$ & $882^{a b c}$ & $24,5^{c}$ & $0,98^{c}$ & $36,2^{a b}$ & $1,45^{a b}$ & $1097^{b c}$ \\
\hline 5 & Buenos Aires & $21^{a}$ & $1,59^{a}$ & $24^{a}$ & $1,84^{\mathrm{a}}$ & $476^{a}$ & $35,8^{a}$ & $1,79^{a}$ & $1036^{a}$ & $27,3^{a b}$ & $1,09^{\mathrm{ab}}$ & $42,5^{a}$ & $1,7^{a}$ & $1483^{a}$ \\
\hline 6 & Montebello & $19,7^{\mathrm{abc}}$ & $1,48^{a}$ & $21,3^{\mathrm{ab}}$ & $1,64^{\mathrm{ab}}$ & $385^{a b}$ & $29,3^{\mathrm{cd}}$ & $1,46^{\mathrm{abcd}}$ & $817^{b c d}$ & - & - & - & - & - \\
\hline 7 & Tactic & $19,1^{\mathrm{abc}}$ & $1,45^{\mathrm{ab}}$ & $21,2^{\mathrm{ab}}$ & $1,63^{a b}$ & $376^{a b}$ & $30,7^{b c d}$ & $1,53^{b c d}$ & $764^{b c d}$ & $25,8^{a b c}$ & $1,03^{a b c}$ & $35,4^{b}$ & $1,42^{b}$ & $1013^{c}$ \\
\hline 8 & $\begin{array}{l}\text { Finca } \\
\text { Las Victorias }\end{array}$ & $20,6^{a}$ & $1,58^{a}$ & $24^{a}$ & $1,85^{a}$ & $474^{a}$ & $34,4^{a b c}$ & $1,72^{\mathrm{ab}}$ & $953^{\mathrm{ab}}$ & $27,6^{a}$ & $1,1^{\mathrm{a}}$ & $41,5^{a}$ & $1,66^{\mathrm{a}}$ & $1378^{\mathrm{ab}}$ \\
\hline 9 & Las Lajas & $20,3^{a b}$ & $1,55^{a}$ & $22,3^{\mathrm{ab}}$ & $1,72^{\mathrm{ab}}$ & $408^{a b}$ & $31,2^{b c d}$ & $1,56^{a b c}$ & $865^{a b c}$ & $25^{c}$ & $1^{c}$ & $36,2^{\mathrm{ab}}$ & $1,45^{\mathrm{ab}}$ & $1083^{b c}$ \\
\hline 10 & San Esteban & $20,5^{a}$ & $1,56^{a}$ & $22,2^{\mathrm{ab}}$ & $1,71^{a b}$ & $406^{a b}$ & $32,7^{a b c}$ & $1,64^{b c d}$ & $801^{b c d}$ & $25,5^{b c}$ & $1,02^{b c}$ & $39,2^{\mathrm{ab}}$ & $1,57^{\mathrm{ab}}$ & $1239^{a b c}$ \\
\hline 11 & Tutul & $18,9^{a b c}$ & $1,46^{a b}$ & $20,7^{b}$ & $1,59^{b}$ & $366^{b c}$ & $33,2^{a b c}$ & $1,66^{b c d}$ & $758^{\text {ncd }}$ & $27,2^{\mathrm{ab}}$ & $1,09^{a b}$ & $38^{a b}$ & $1,52^{a b}$ & $1191^{\mathrm{abc}}$ \\
\hline 12 & Los Alpes & $19,7^{\mathrm{abc}}$ & $1,48^{a}$ & $22,2^{\mathrm{ab}}$ & $1,71^{a b}$ & $413^{a b}$ & $33,1^{a b c}$ & $1,65^{a b c}$ & $838^{\text {abcd }}$ & $26,9^{\mathrm{ab}}$ & $1,08^{a b}$ & $39,2^{a b}$ & $1,57^{a b}$ & $1237^{a b c}$ \\
\hline \multirow[t]{3}{*}{13} & Yucul & $19,4^{\mathrm{abc}}$ & $1,49^{a}$ & $23^{a b}$ & $1,77^{a b}$ & $434^{\mathrm{ab}}$ & $32,2^{a b c}$ & $1,61^{\mathrm{cd}}$ & $696^{\text {cd }}$ & $25,9 \mathrm{abc}$ & $1,04^{\mathrm{abc}}$ & $39^{\mathrm{ab}}$ & $1,56^{a b}$ & $1220^{a b c}$ \\
\hline & Moyenne & 19,5 & 1,5 & 21,8 & 1,68 & 397 & 31,9 & 1,59 & 833 & 26,1 & 1,04 & 38,4 & 1,53 & 1201 \\
\hline & Écart-type & 4,09 & 0,46 & 5,64 & 0,43 & 184,4 & 6,23 & 0,31 & 315,1 & 2,87 & 0,11 & 7,54 & 0,3 & 452,9 \\
\hline
\end{tabular}

$\mathrm{D}$ : diamètre à $1,30 \mathrm{~m}$; $\mathrm{H}$ : hauteur totale ; $\mathrm{n}$ : nombre d’individus ; Amah : accroissement moyen annuel en hauteur ;

Amad : accroissement moyen annuel en diamètre $; \mathrm{G}:$ surface terrière.

a, ab, ... : classification des groupes non significativement différents à $5 \%$ selon la méthode de Holm.

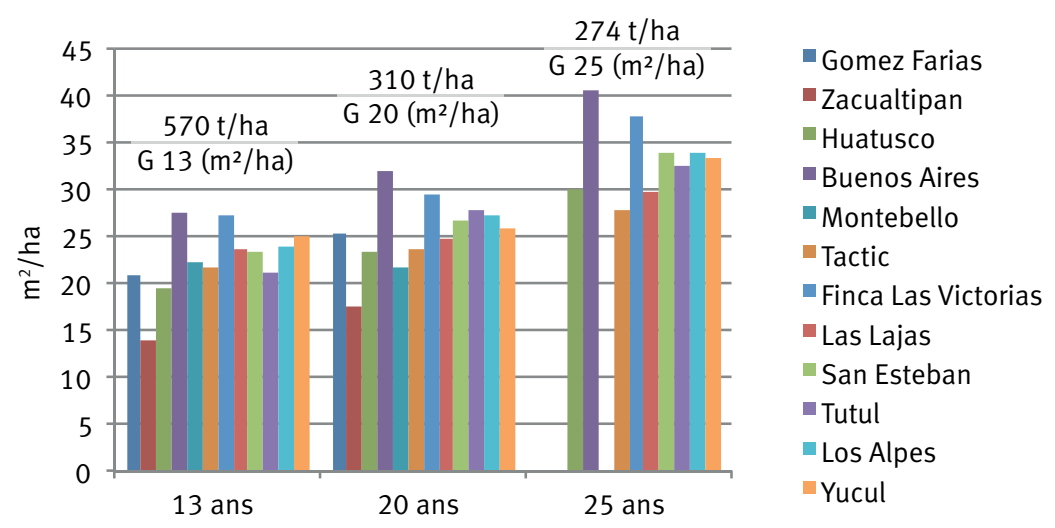

Figure 4.

Surface terrière de chaque provenance de Liquidambar styraciflua à Mandraka à différents âges.

\section{Croissance de Liquidambar styraciflua à Mandraka comparée aux espèces exotiques courantes à Madagascar et au Liquidambar des autres essais de provenances du Cfi}

Comparé aux autres espèces de plantation à Madagascar (Eucalyptus robusta, E. camaldulensis, Pinus patula et $P$. kesiya), à âges équivalents et dans des conditions écologiques similaires (ou proches), l'accroissement moyen annuel en hauteur à 13 ans du liquidambar de l'essai de provenances à Mandraka est largement supérieur (tableau IV). Quant à la croissance en diamètre à 13 ans, celle du liquidambar de l'essai à Mandraka se rapproche des espèces du genre Pinus. En revanche, la croissance en diamètre du liquidambar est inférieure à celle d'E. camaldulensis (tableau IV). L'accroissement annuel moyen en hauteur et en diamètre de $L$. styraciflua à 25 ans de l'essai de provenances de Mandraka est largement supérieur à celui de la station forestière de Mandraka gérée par le Département des eaux et forêts de l'École supérieure des sciences agronomiques. Cela est dû à l'absence de soins syl- 


\section{Tableau IV.}

Comparaison de la croissance de Liquidambar styraciflua de l'essai de provenances de Mandraka avec les espèces de plantation les plus courantes dans les mêmes conditions écologiques et à âges équivalents à Madagascar.

\begin{tabular}{|c|c|c|c|c|c|}
\hline Espèce & $\begin{array}{c}\text { Âge } \\
\text { (années) }\end{array}$ & $\underset{(m)}{\operatorname{Amah}}$ & $\begin{array}{c}\text { Âge } \\
\text { (années) }\end{array}$ & $\begin{array}{l}\text { Amad } \\
(\mathrm{cm})\end{array}$ & \multirow{3}{*}{$\begin{array}{l}\text { BD Drfp + Inventaire de } 2011 \\
+ \text { TP Essa-Forêts }\end{array}$} \\
\hline de provenances à Mandraka & 20 & - & 20 & 1,59 & \\
\hline & 25 & 1 & 25 & 1,53 & \\
\hline $\begin{array}{l}\text { Pinus patula (zone B du Parv: } \\
\text { versant Est du Haut Plateau) }\end{array}$ & 14 & 0,74 & 11 & 1,23 & $\begin{array}{l}\text { RAKOTONDRAOELINA (1994) ; CHAUVET (1969) } \\
\text { in RAMAMONJISOA (1999) }\end{array}$ \\
\hline Pinus kesiya à Manjakatompo & 12 & 0,71 & 11 & 1,42 & RANDRIANJAFY (1993) \\
\hline $\begin{array}{l}\text { Eucalyptus robusta } \\
\text { (arbre plus à Périnet-Mantadia) }\end{array}$ & 16 & 1 & ND & ND & \\
\hline
\end{tabular}

Amah : accroissement moyen annuel en hauteur; Amad : accroissement moyen annuel en diamètre ; ND : non disponible ; Parv : Projet d'appui au reboisement villageois ; BD : base de données ; TP : travaux pratiques, Essa : École supérieure des sciences agronomiques.

vicoles et à la forte densité de peuplement (2 140 tiges/ha). Cependant, l'accroissement annuel moyen en hauteur et en diamètre de L. styraciflua de la station forestière de Mandraka, respectivement de 0,9 $\mathrm{m}$ et de 1,04 cm à 25 ans, même en l'absence de soins sylvicoles et avec une forte densité de population, confirme l'adaptation et la potentialité élevée en croissance de cette espèce sur ce site. Comparé aux autres essais du Cfi, seul l'essai de provenances de $L$. styraciflua à Iguaçu (Brésil) dépasse celui de Mandraka aussi bien en hauteur qu'en diamètre. Les autres essais de Xalapa (Mexique), de Paraguaçu et de Agudos (Brésil) ont une croissance moyenne inférieure à celle de Mandraka, aux quatre âges considérés (tableau V).

\section{Variabilité intraspécifique de la croissance de Liquidambar styraciflua à Mandraka}

Les provenances testées à Mandraka n'ont pas les mêmes potentialités de croissance. Les analyses de la variance ont montré qu'au seuil entre 0,1 et $5 \%$ l'effet provenance est significatif sur la croissance moyenne en hauteur, en diamètre et en surface terrière de L. styraciflua (tableau VI). L'effet bloc est statistiquement significatif vis-à-vis de la valeur moyenne de la hauteur des provenances à 13 ans. Les arbres des blocs I, III et IV ont des hauteurs significativement plus élevées que ceux du bloc II. Cette différence est surtout due à la fertilité du sol du bloc II et à la concurrence en lumière. En effet, ce bloc se trouve sur la partie où la pente du terrain est très forte, entraînant le lessivage des éléments minéraux, et conduisant probablement à une fertilité moindre. En revanche, l'effet bloc n'est pas significatif à 25 ans probablement par l'effet des éclaircies sélectives. L'effet d'interac-

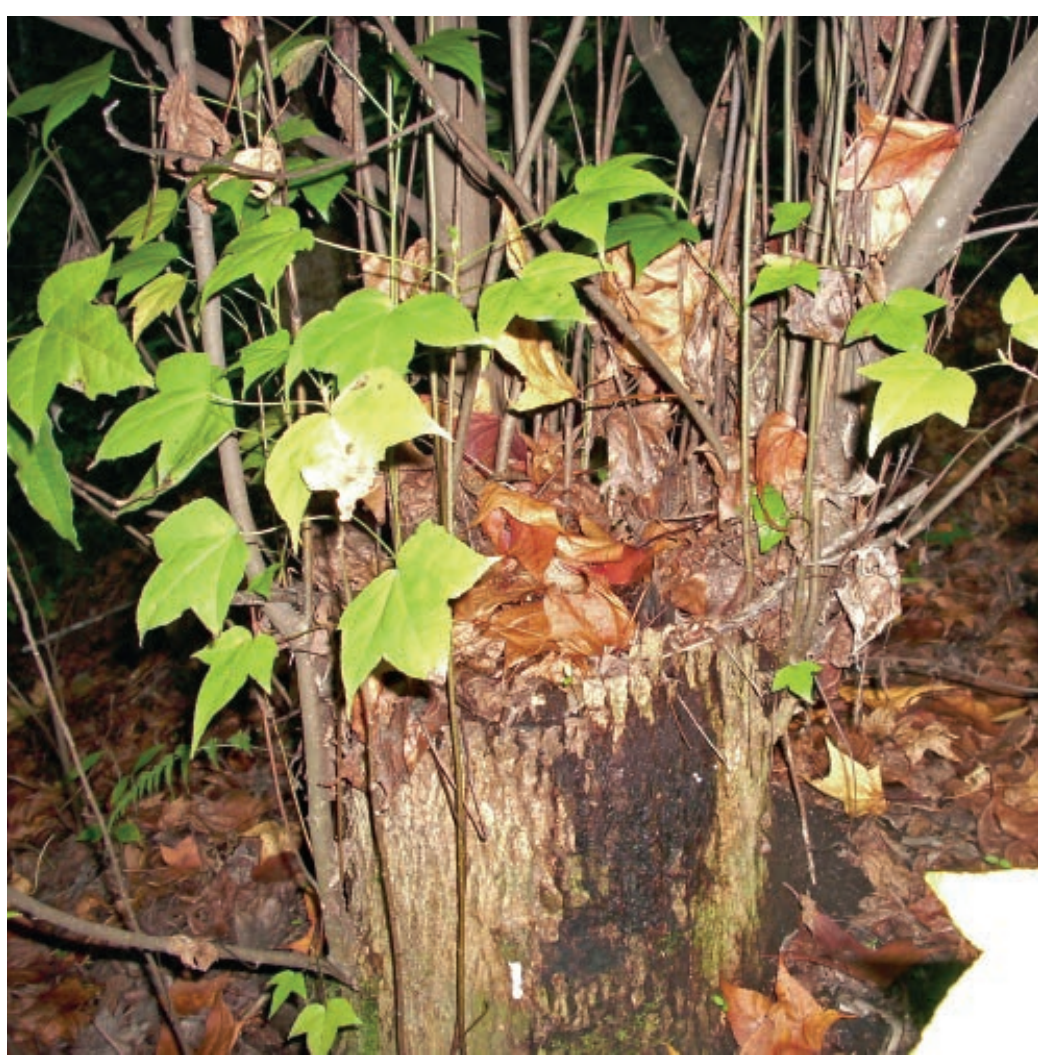

Photo 2.

Rejets de souche de Liquidambar styraciflua de l'essai de provenances à Mandraka. 
Tableau V.

Comparaison de la croissance de Liquidambar styraciflua à Mandraka par rapport aux autres essais de provenances du Cfi

\begin{tabular}{|c|c|c|c|c|c|c|c|c|c|c|c|}
\hline Paramètre & Localité & Âge & Min & Max & Moyenne & $\begin{array}{l}\text { Écart- } \\
\text { type }\end{array}$ & $\begin{array}{l}\text { Coordonnées } \\
\text { géographiques }\end{array}$ & $\begin{array}{l}\text { Température } \\
\left({ }^{\circ} \mathrm{C}\right)\end{array}$ & $\begin{array}{l}\text { Pluviométrie } \\
(\mathrm{mm})\end{array}$ & $\begin{array}{l}\text { Altitude } \\
\text { (m) }\end{array}$ & Référence \\
\hline $\mathrm{D}_{1,30}(\mathrm{~cm})$ & $\begin{array}{l}\text { Mandraka } \\
\text { Agudos }\end{array}$ & $\begin{array}{l}9 \\
9\end{array}$ & $\begin{array}{l}13,8 \\
12,5\end{array}$ & $\begin{array}{l}18,1 \\
16,5\end{array}$ & $\begin{array}{l}16,4 \\
14,5\end{array}$ & $\begin{array}{l}1,3 \\
1,3\end{array}$ & $\begin{array}{l}18^{\circ} 54^{\prime} \mathrm{S} 47^{\circ} 55^{\prime} \mathrm{E} \\
22^{\circ} 19^{\prime} 05^{\prime \prime} \mathrm{N} 48^{\circ} 52^{\prime} 00 \mathrm{~W}\end{array}$ & $\begin{array}{l}17,5 \\
21,1\end{array}$ & $\begin{array}{r}2300 \\
550\end{array}$ & $\begin{array}{l}1200 \\
1300\end{array}$ & SHIMIZU, SPIR (1999) \\
\hline $\mathrm{H}(\mathrm{m})$ & $\begin{array}{l}\text { Mandraka } \\
\text { Iguaçu }\end{array}$ & $\begin{array}{l}11 \\
11\end{array}$ & $\begin{array}{l}13,5 \\
18,9\end{array}$ & $\begin{array}{l}19,4 \\
21,6\end{array}$ & $\begin{array}{l}16,4 \\
20,4\end{array}$ & $\begin{array}{l}2,0 \\
0,9\end{array}$ & $\begin{array}{l}18^{\circ} 54^{\prime} \mathrm{S} 47^{\circ} 55^{\prime} \mathrm{E} \\
25^{\circ} 41^{\prime} 55^{\prime \prime} \mathrm{S} 54^{\circ} 26^{\prime} 13^{\prime \prime} \mathrm{W}\end{array}$ & $\begin{array}{l}17,5 \\
15 \text { à } 19\end{array}$ & $\begin{array}{r}2300 \\
1250 \text { à } 2500\end{array}$ & $\begin{array}{r}1200 \\
196\end{array}$ & SHIMIZU, SPIR (2002) \\
\hline $\mathrm{D}_{1,30}(\mathrm{~cm})$ & $\begin{array}{l}\text { Mandraka } \\
\text { Iguaçu }\end{array}$ & $\begin{array}{l}11 \\
11\end{array}$ & $\begin{array}{l}16,4 \\
21,0\end{array}$ & $\begin{array}{l}21,3 \\
27,7\end{array}$ & $\begin{array}{l}19,1 \\
23,5\end{array}$ & $\begin{array}{l}1,6 \\
2,3\end{array}$ & $\begin{array}{l}18^{\circ} 54^{\prime} \mathrm{S} 47^{\circ} 55^{\prime} \mathrm{E} \\
25^{\circ} 41^{\prime} 55^{\prime \prime} \mathrm{S} 54^{\circ} 26^{\prime} 13^{\prime \prime} \mathrm{W}\end{array}$ & $\begin{array}{l}17,5 \\
15 \text { à } 19\end{array}$ & $\begin{array}{r}2300 \\
1250 \text { à } 2500\end{array}$ & $\begin{array}{r}1200 \\
196\end{array}$ & SHIMIZU, SPIR (2002) \\
\hline $\mathrm{D}_{1,30}(\mathrm{~cm})$ & $\begin{array}{l}\text { Xalapa } \\
\text { Mandraka }\end{array}$ & $\begin{array}{l}16 \\
16\end{array}$ & $\begin{array}{l}13,7 \\
18,6\end{array}$ & $\begin{array}{l}17,4 \\
25,8\end{array}$ & $\begin{array}{l}15,6 \\
23,4\end{array}$ & $\begin{array}{l}1,4 \\
2,2\end{array}$ & $\begin{array}{l}19^{\circ} 34^{\prime} 08^{\prime \prime} \mathrm{N} 19^{\circ} 55^{\prime} 55^{\prime \prime} \mathrm{W} \\
18^{\circ} 54^{\prime} \mathrm{S} 47^{\circ} 55^{\prime} \mathrm{E}\end{array}$ & $\begin{array}{l}19,8 \\
17,5\end{array}$ & $\begin{array}{l}1500 \\
2300\end{array}$ & $\begin{array}{l}1420 \\
1200\end{array}$ & $\begin{array}{l}\text { MENDIZABAL-HERNANDEZ } \\
\text { et al., (2009) }\end{array}$ \\
\hline $\mathrm{D}_{1,30}(\mathrm{~cm})$ & $\begin{array}{l}\text { Paraguaçu } \\
\text { Mandraka }\end{array}$ & $\begin{array}{l}19 \\
19\end{array}$ & $\begin{array}{l}11,6 \\
28,8\end{array}$ & $\begin{array}{l}27,29 \\
33,7\end{array}$ & $\begin{array}{l}20,4 \\
31,3\end{array}$ & $\begin{array}{l}5,1 \\
1,8\end{array}$ & $\begin{array}{l}22^{\circ} 25^{\prime} \mathrm{S} 50^{\circ} 35^{\prime} \mathrm{W} \\
18^{\circ} 54^{\prime} \mathrm{S} 47^{\circ} 55^{\prime} \mathrm{E}\end{array}$ & $\begin{array}{l}16,0 \\
17,5\end{array}$ & $\begin{array}{l}1131 \\
2300\end{array}$ & $\begin{array}{r}490 \\
1200\end{array}$ & SEBBENN et al., (2007) \\
\hline
\end{tabular}

tion bloc croisé à la provenance n'est significatif que pour la hauteur à 13 ans. En d'autres termes, les effets de la disponibilité en lumière, de la fertilité et de la topographie sur la hauteur ne peuvent être observés qu'à 13 ans.

La comparaison deux à deux des moyennes des hauteurs, des diamètres et des surfaces terrières à 13, 20 et 25 ans (tableau III) montre que les croissances des provenances Buenos Aires et Finca Las Victorias est significativement la plus élevée. À l'inverse, les provenances Zacualtipan et Huatusco présentent les croissances les plus faibles. Ce classement est confirmé par la surface terrière moyenne par provenance (figure 2). Par conséquent, à Mandraka, les prove- nances Buenos Aires et Finca Las Victorias sont les plus productives en volume de bois par rapport aux autres.

Les valeurs de l'héritabilité du diamètre, de la hauteur et de la surface terrière allant de 0,60 à 0,87 montrent que ces caractères sont sous contrôle génétique (tableau VI). Il est donc envisageable de réaliser des sélections au niveau des provenances en se basant sur la valeur moyenne de la hauteur, du diamètre et de la surface terrière des individus qui les composent. À cet effet, une sélection à 25 ans permettra d'envisager un gain génotypique de $8 \%, 11 \%$ et $25 \%$ respectivement pour la hauteur, le diamètre et la surface terrière.

\section{Tableau VI.}

Comparaison de la moyenne de la hauteur et du diamètre de Liquidamba styraciflua à Mandraka suivant les provenances, les blocs et leurs interactions.

\begin{tabular}{|c|c|c|c|c|c|c|c|c|c|}
\hline Paramètre & $\begin{array}{l}\text { Source de } \\
\text { variabilité }\end{array}$ & ddl & $\begin{array}{l}\text { P-value } \\
\text { à } 13 \text { ans } \\
\text { (1999) }\end{array}$ & $\mathrm{h}^{2}{ }_{\mathrm{G}}$ & ddl & $\begin{array}{l}\text { P-value } \\
\text { à } 20 \text { ans } \\
(2006)\end{array}$ & $h^{2}{ }_{G}$ & ddl & $\begin{array}{l}\text { P-value } \\
\text { à } 25 \text { ans } \\
\text { (2011) }\end{array}$ \\
\hline \multirow{3}{*}{ Diamètre $\emptyset_{1,30}$} & Bloc & 3 & 0,339 & \multirow[t]{3}{*}{0,81} & 3 & 0,327 & \multirow[t]{3}{*}{0,76} & 3 & 0,811 \\
\hline & Provenance & 11 & $0,001^{\star \star}$ & & 11 & $0,001^{\star \star}$ & & 8 & $0,011^{*}$ \\
\hline & Bloc $x$ provenance & 33 & 0,435 & & 33 & 0,487 & & 24 & 0,252 \\
\hline \multirow[t]{3}{*}{ Surface terrière (cm²/arbre) } & Bloc & 3 & 0,367 & \multirow[t]{3}{*}{0,81} & 3 & 0,432 & \multirow[t]{3}{*}{0,76} & 3 & 0,587 \\
\hline & Provenance & 11 & $<0,001^{\star \star \star}$ & & 11 & $<0,001^{\star \star \star}$ & & 8 & $0,001^{\star \star}$ \\
\hline & Bloc $x$ provenance & 33 & $0,001^{\star \star}$ & & 33 & 0,528 & & 24 & 0,641 \\
\hline \multirow[t]{3}{*}{ Hauteur (m) } & Bloc & 3 & $<0,001^{\star \star \star}$ & \multirow[t]{3}{*}{0,87} & - & - & \multirow[t]{3}{*}{ - } & 3 & 0,830 \\
\hline & Provenance & 8 & $<0,001^{\star \star \star}$ & & - & - & & 8 & $0,001^{\star \star \star}$ \\
\hline & Bloc $x$ provenance & 24 & $0,001^{\star \star}$ & & - & - & & 24 & 0,222 \\
\hline
\end{tabular}




\section{Discussion et recommandations}

\section{Adaptation et croissance de Liquidambar styraciflua}

La croissance rapide en hauteur de $L$. styraciflua dans l'essai de provenances à Mandraka au jeune âge et sa diminution progressive avec l'âge indiquent que cette espèce est pionnière à tempérament héliophile. La comparaison de la croissance de Liquidambar aux autres espèces exotiques courantes à Madagascar (Eucalyptus robusta, E. camaldulensis, Pinus patula et $P$. kesiya) montre que $L$. styraciflua est une espèce prometteuse en matière de production de bois et le site d'essai de Mandraka convient bien à son développement.

La bibliographie existante pour cette espèce (WRIGHT, CUNNINGHAM, 2008 ; ORWA et al., 2009) conforte les résultats obtenus lors de la comparaison des sites d'autres pays avec celui de Madagascar. Le site d'Iguaçu, avec une température moyenne de $19{ }^{\circ} \mathrm{C}$ et une pluviosité annuelle de $2500 \mathrm{~mm}$ bien répartie toute l'année (SHIMIZU, SPIR, 2002), est celui où les conditions sont optimales pour la croissance de $L$. styraciflua. La différence principale entre Iguaçu et Mandraka réside dans la constance des précipitations. En effet, le site de Mandraka connaît un déficit au plan hydrique d'août à octobre et d'avril à juin contrairement au site d'Iguaçu.

Le site de Mandraka constitue ainsi un site potentiel pour une forte croissance de L. styraciflua étant donné que les conditions environnementales à Mandraka sont semblables à celles de son aire naturelle de distribution. En effet, se situant sur le versant Est de la première falaise orientale de Madagascar, des vents frais et humides passent habituellement à Mandraka. La pluviosité et la température moyennes annuelles sont respectivement de $2300 \mathrm{~mm}$ et $17{ }^{\circ} \mathrm{C}$. Ces constats confirment les résultats avancés par CARTER et HUGHES (1984) sur la potentialité de la croissance de l'espèce dans les zones tropicales fraîches.

\section{Variabilité intraspécifique de la croissance de Liquidambar styraciflua}

Des différences significatives de la moyenne de la hauteur et du diamètre suivant les provenances ont été observées dans tous les autres essais de provenances de L. styraciflua au Brésil et au Mexique, quel que soit l'âge (SHIMIZU, SPIR, 1999, 2002 ; MENDIZABAL-HERNANDEZ et al., 2009 ; SEBBENN et al., 2007). Il existe donc des provenances qui s'adaptent mieux aux sites où elles sont introduites.

Selon SHIMIZU et SPIR (1999, 2002), les provenances issues de latitudes au sud de $15^{\circ} \mathrm{N}$ avec une température moyenne annuelle supérieure à $18{ }^{\circ} \mathrm{C}$ s'adaptent mieux en termes de croissance par rapport aux autres provenances de latitude et de températures différentes, quand elles sont plantées dans d'autres sites hors zone de distribution. SEBBENN et al. (2007) ont par ailleurs rapporté que la croissance des arbres dans les sites d'introduction peut dépendre de la différence entre la pluviométrie du site d'introduction et celle de la région d'origine. Pour le cas de Mandraka, l'effet combiné de la température, de la pluviosité annuelle, de la latitude et de l'altitude a conditionné l'adaptation de chaque provenance. En effet, celles de Buenos Aires et Finca Las Victorias présentent des conditions climatiques d'origine semblables à celles de Mandraka, avec une altitude comprise entre 900 et $1300 \mathrm{~m}$, une pluviosité annuelle de l'ordre de $2000 \mathrm{~mm}$ et une température moyenne annuelle supérieure à $19{ }^{\circ} \mathrm{C}$. Les autres provenances se trouvent dans des conditions différentes des précédentes et peuvent exceller dans d'autres sites similaires à leurs zones de distribution ; d'où l'intérêt de réaliser des essais multisites. Dans notre étude, les provenances se trouvant au sud de la latitude $15,5^{\circ} \mathrm{N}$ dans leurs aires naturelles de distribution ont une faculté d'adaptation en croissance élevée quand elles sont introduites dans des sites dont les conditions climatiques, topographiques et édaphiques sont semblables à celles de Mandraka.

La classification des provenances de $L$. styraciflua se base ici sur le seul site de Mandraka. Il aurait été intéressant de réaliser des essais multisites afin de pouvoir évaluer l'effet site et l'interaction génotype croisé au site, et de mieux estimer l'héritabilité. Pour le cas de Madagascar, outre le site de Mandraka, deux autres sites avec des conditions écologiques et topographiques différentes avaient été choisis en même temps que Mandraka. Il s'agit du site de Morarano (930 m d'altitude, à l'Est d'Antananarivo, à $15 \mathrm{~km}$ d'Androfia dans le périmètre de plantation de la société Fanalamanga) et du site d'Androfia (980 m d'altitude, à l'Est d'Antananarivo, et à 30 km au Nord de Moramanga). L'essai de plantation à Androfia n'a pas eu lieu à cause des contestations des propriétaires de terrain et celui du Morarano n'a pas survécu longtemps du fait des feux de brousse. Néanmoins, l'essai de Mandraka comporte quatre blocs dont les conditions environnementales sont différentes s'agissant de la fertilité du sol, de la topographie, de l'exposition au vent et de l'ensoleillement. C'est pour cela que des différences ont été observées entre les hauteurs des arbres dans chaque bloc à 13 ans. Ces variations restent toutefois plus faibles par rapport à celles des deux autres sites initialement retenus avec celui de Mandraka. Nos résultats sont exploitables pour des régions dont les conditions environnementales sont proches de celles de Mandraka. Ainsi, dans l'objectif de produire des individus à croissance rapide, il est possible de réaliser en premier lieu des sélections sur la base des meilleures provenances. Des sélections individuelles peuvent être réalisées en deuxième lieu dans les meilleures provenances. Pour des régions dont les conditions écologiques sont similaires à celles de Mandraka, les provenances de Buenos Aires et de Finca Las Victorias sont recommandées pour leur croissance rapide. Dans ce cas, l'essai de provenances à Mandraka peut être utilisé comme verger à graines à défaut de ne pas avoir pu réaliser des clonages des meilleurs phénotypes issus de meilleures provenances.

L'effet bloc n'est pas significatif et l'interaction x provenance n'est significative que pour la hauteur et à 13 ans. La variabilité des caractéristiques dendrométriques de 


\section{Conclusion}

L. styraciflua n'est donc pas seulement d'origine génétique, il y a aussi l'effet de l'environnement. En effet et en dépit des observations faites sur le terrain, il se pourrait que la variabilité de ces conditions ne soit pas suffisamment élevée pour mettre en évidence des différences statistiquement significatives. C'est pour cela que l'effet d'interaction n'a été observé qu'au jeune âge. Pour une meilleure sélection des provenances dans le futur, il est recommandé de réaliser des essais de descendances multisites. Dans ce cas, l'essai de provenances à Mandraka peut servir de source semencière afin de constituer ces essais. En outre, l'évaluation des descendants permettra d'avoir une idée de la valeur des parents (arbres mères) en croisement.

Pour le cas de l'essai à Mandraka, il est recommandé de garder le nombre de provenances afin d'avoir une base génétique suffisamment large pour l'amélioration de l'espèce, du fait que les autres provenances peuvent exprimer leur pleine potentialité dans d'autres sites. Dans ce sens, après avoir réalisé des essais comparatifs de descendances multisites et vérifié la potentialité de chaque provenance, il est recommandé d'introduire plus massivement les meilleures d'entre-elles et transformer les plantations effectuées en parcelles semencières pour la diffusion de semences améliorées de L. styraciflua. Cependant, il faut toujours choisir les provenances en fonction du site de reboisement. Ainsi, il devrait y avoir plusieurs sites semenciers avec des provenances différentes.

Les travaux d'éclaircie menés dans l'essai de la Mandraka ont montré que le $L$. styraciflua réagit bien aux éclaircies. Ce qui montre l'importance alors de réaliser des soins sylvicoles pour les futures plantations de L. styraciflua à Madagascar. Les taux et le nombre d'éclaircies à Mandraka sont un exemple concret de la gestion d'une plantation de $L$. styraciflua pour une exploitation à vocation de production de bois.

\section{Estimation de l'héritabilité}

L'expérimentation de L. styraciflua à Mandraka n'est pas la plus adaptée pour une estimation de l'héritabilité des caractères de croissance (FALCONER et MACKAY, 1996) considérés ici, pour les principales raisons : le nombre de provenance variable d'un bloc à une autre ; l' essai sur un seul site ; la présence non contrôlée d'individus plein frères et demi-frères. Cela a pour conséquence de diminuer la variance totale des caractères étudiés et de surestimer l'héritabilité. Mais selon NANSON (1970) la méthode retenue est la plus adéquat à l'expérimentation à Mandraka.
Cette étude a permis de montrer la potentialité de croissance en hauteur et en diamètre de Liquidambar styraciflua à Mandraka. L'accroissement moyen annuel en hauteur et en diamètre à 25 ans est respectivement de $1 \mathrm{~m}$ et de $1,5 \mathrm{~cm}$. À l'exception de l'espèce Eucalyptus camaldulensis, la croissance de $L$. styraciflua à Mandraka est plus rapide que celle des autres espèces introduites à Madagascar (Eucalyptus robusta, Pinus patula et $P$. kesiya) dans les mêmes conditions environnementales. En se basant sur les paramètres de croissance et par rapport aux autres sites d'essai de provenances de $L$. styraciflua du Cfi, celui de Mandraka tient le deuxième rang après l'essai d'Iguaçu (Brésil).

De par sa croissance, le comportement de L. styraciflua correspond à celui d'une espèce pionnière à tempérament héliophile. Cette étude a mis en évidence qu'il est possible d'exploiter l'espèce en tant que bois d'œuvre avec une révolution de 20 ans. Du point de vue de la variabilité, l'effet provenance est significatif pour la hauteur, le diamètre et la surface terrière. Les provenances Buenos Aires et Finca Las Victorias sont les plus performantes quand elles sont introduites dans des sites comme de la Mandraka. Cela n'exclut pas que les autres provenances peuvent exprimer leurs potentialités dans d'autres sites, d'où l'intérêt de la réalisation d'essais multisites. Ces essais multisites sont aussi d'une importance capitale du fait de la différence des moyennes des hauteurs seulement observée au jeune âge.

L'estimation de l'héritabilité a démontré que la hauteur, la circonférence et la surface terrière sont sous fort contrôle génétique $\left(\mathrm{h}_{\mathrm{G}}^{2}\right.$ entre 0,60 et 0,87$)$. Ces trois caractères peuvent ainsi être utilisés en sélection pour l'amélioration de l'espèce et espérer un gain de $8 \%$ pour la hauteur, $11 \%$ pour le diamètre et $25 \%$ pour la surface terrière dans le cas de l'essai de Mandraka.

Les résultats de ce travail se sont basés principalement sur les valeurs de croissance en hauteur, en diamètre et en surface terrière de L. styraciflua. Ces données sont certes d'une importance capitale mais elles sont insuffisantes pour conclure sur la potentialité de l'espèce à être domestiquée et surtout à répondre aux besoins en bois des Malgaches en complément des espèces déjà utilisées. Des études complémentaires ont été menées sur la faculté de multiplication de l'espèce ainsi que les propriétés de son bois. Les résultats prochainement publiés devraient compléter ces premiers présentés ici et permettre de savoir par exemple si certaines propriétés du bois peuvent être également de bons critères pour une sélection multi-critères. 


\section{Références bibliographiques}

ALDEN A., 1995. Hardwoods of North America. Washington, DC, USA, US Department of Agriculture, Forest Service, Forest Products Laboratory, General Technical Report FPL-GTR-83, 136 p.

AMBROISE C., 2007. Tests d'hypothèses multiples. Laboratoire Statistique et Génome, Umr Cnrs 8071, France, 28 diapositives.

CARTER P. M., HUGHES C. E., 1986. Liquidambar styraciflua L. A species of potential for the tropics. Commonwealth Forestry Review, 63 (3): 207-216.

FALCONER D. S., MACKAY T. F. C., 1996. Introduction to Quantitative Genetics. ${ }^{\text {th }}$ edn. Londres, Royaume-Uni, Longman Group, 464 p.

FAO, 2007. Situation des forêts du monde 2007. Rome, Italie, Fao, Département forêts, 351 p.

FOFIFA, 1990. Introduction d'espèces exotiques à Madagascar. Rapport de synthèse. Antananarivo, Madagascar, Fofifa, Département de recherches forestières et piscicoles, tome I, non paginé.

MATTOS P. P. D., PEREIRA J. C. D., SCHAITZA A. G., CARVALHO P. E. R., 2001. Caracteristicas de madeira de Liquidambar styraciflua. Colombo, Brésil, Embrapa Florestas, Circular Téchnica, 49, 4 p.

MENDIZABAL-HERNANDEZ L. D. C., RAMIREZ J. M., ALBALANDA J., RAMIREZ-GARCIA E. O., RAMIREZ-GARCIA H., 2009. Alternativas de uso de una prueba genética de Liquidambar styraciflua L. Foresta Veracruzana, 11 (2): 22-32.

MINISTÈRE DE L'ENVIRONNEMENT, DES EAUX ET FORÊTS, 1998. Décret $n^{\circ}$ 98-782 du 16 septembre 1998 relatif au régime de l'exploitation forestière. Antananarivo, Madagascar, Ministère de l'Environnement, des Eaux et Forêts, 19 p. MOUCHIROUD D., 2003. Mathématique : outils pour la biologie. Chapitre 9 : Analyse de variance. Lyon, France, Université Claude-Bernard 1, 11 p.

NANSON A., 1970. L'héritabilité et le gain d'origine génétique dans quelques types d'expériences. Sylvae Genetica, 19 (4) : 113-121.

ORWA C., MUTUA A., KINDT R., JAMNADASS R., ANTHONNY S., 2009. Agroforestree Database: a tree reference and selection guide, version 4.0. Nairobi, Kenya, World Agroforestry Centre.

R DEVELOPMENT CORE TEAM., 2012. R: A language and environment for statistical computing. Vienne, Autriche, R Foundation for Statistical Computing. http://www.R-project.org/.

RAKOTONDRAOELINA H., 1994. Test de descendances de Pinus patula Schield et Doppe de Sambilahy-Ampamaherana. Mémoire de diplôme d'études approfondies, Université d'Antananarivo, Madagascar, 57 p.

RAKOTONDRAOELINA H. A., RAKOTOVAO G., 2005. Le «Copalme d'Amérique ", une belle espèce feuillue à croissance rapide pour la production de bois d'œuvre. Ambatobe, Madagascar, Fofifa-Drfp, 4 p.
RAMAMONJISOA B. S., 1999. Rapport de compilation et d'analyse des données existantes sur le secteur des plantations forestières de Madagascar : État des plantations villageoises et familiales malgaches d'aujourd'hui. Rome, Italie, CE-Fao, 28 p.

RANDRIANJAFY H., 1993. Production et aménagement des taillis d'eucalyptus à courte rotation : le cas des peuplements d'Eucalyptus robusta Smith, à vocation de bois d'énergie, sur les hautes terres centrales de Madagascar. Zurich, Suisse, École polytechnique fédérale de Zurich, $263 \mathrm{p}$.

SABISTINA B., 2010. Gum : après les paysagistes, il séduit les industriels. Le Bois International, 8 : 15-16.

SAPORTA G., 2006. Probabilités, analyse des données et statistique. Paris, France, Éditions Technip, 622 p.

SEBBENN A. M., ARANTES F., BOAS O. V., FREITAS M. L. M., 2007. Performance of 19-year-old provenances of Liquidambar styraciflua in Paraguaçu Paulista, São Paulo. Crop Breeding and Applied Biotechnology, 7 : 381-386.

SHIMIZU J. Y., SPIR I. H. Z., 1999. Avaliação de procedências e progênies de Liquidambar da América central, do México e dos Estados Unidos, em Agudos, Brasil. Boletim de Pesquisa Florestal, 39: 93-108.

SHIMIZU J. Y., SPIR I. H. Z., 2002. Produtividade de madeira de liquidambar de diferentes procedências em Quedas do Iguaçu, Paraná. Boletim de Pesquisa Florestal, 44: 3-12.

WRIGHT J., CUNNINGHAM M., 2008. Sweetgum plantations for sawtimber, energy, pulp, and other uses. Forest Landowner Magazine, 67 (3): 26-28. 